Published in final edited form as:

MCN Am J Matern Child Nurs. 2016 ; 41(4): 237-243. doi:10.1097/NMC.0000000000000244.

\title{
Milk flow rates from bottle nipples used after hospital discharge
}

Britt Frisk Pados, PhD, RN, NNP-BC,

Assistant Professor, University of North Carolina at Chapel Hill School of Nursing

Jinhee Park, PhD, RN,

Assistant Professor, Boston College School of Nursing

Suzanne M. Thoyre, PhD, RN, FAAN,

Francis Hill Fox Distinguished Term Professor, University of North Carolina at Chapel Hill School of Nursing

Hayley Estrem, PhD, RN, and

Postdoctoral Associate, Duke University School of Nursing

\section{W. Brant Nix, BMET, BA}

Medical Laboratory Technologist \& Lab Manager, Biobehavioral Laboratory, University of North Carolina at Chapel Hill School of Nursing

\section{Abstract}

Purpose-To test the milk flow rates and variability in flow rates of bottle nipples used after hospital discharge.

Study Design and Methods-Twenty-six nipple types that represented 15 common brands as well as variety in price per nipple and store location sold (e.g., Babies R' Us, Walmart, Dollar Store) were chosen for testing. Ten of each nipple type ( $n=260$ total) were tested by measuring the amount of infant formula expressed in one minute using a breast pump. Mean milk flow rate ( $\mathrm{mL} /$ $\min )$ and coefficient of variation $(\mathrm{CV})$ were calculated. Flow rates of nipples within brand were compared statistically.

Results-Milk flow rates varied from $1.68 \mathrm{~mL} / \mathrm{min}$ for the Avent Natural Newborn Flow to $85.34 \mathrm{~mL} / \mathrm{min}$ for the Dr. Brown's Standard Y-cut. Variability between nipple types also varied widely, from .03 for the Dr. Brown's Standard Level 3 to .37 for MAM Nipple 1 Slow Flow.

Clinical Implications-The extreme range of milk flow rates found may be significant for medically fragile infants being discharged home who are continuing to develop oral feeding skills. The name of the nipple does not provide clear information about the flow rate to guide parents in decision-making. Variability in flow rates within nipples of the same type may complicate oral feeding for the medically fragile infant who may not be able to adapt easily to change in flow rates. Both flow rate and variability should be considered when guiding parents to a nipple choice.

Conflicts of Interest: None

Disclosure: Data for the six Dr. Brown's nipples that are included in this paper were previously published in a paper entitled Milk flow rates from bottle nipples used for feeding hospitalized infants. This paper is in press and has been published in advance online. 


\section{Keywords}

Infant; Bottle Feeding; Feeding Methods; Swallowing

Milk flow rate is the rate at which milk moves from the bottle nipple into the infant's mouth during bottle-feeding. Milk flow has been found to contribute to respiratory disruption during feeding (al-Sayed, Schrank, \& Thach, 1994; Mathew, 1991). Since there are common anatomical structures between swallowing and breathing, respiration must be paused momentarily during the swallow to prevent aspiration of liquid (Barlow, 2009). As milk flow increases, the rate of swallowing must increase, and therefore, the degree of respiratory interruption increases (al-Sayed, et al., 1994). If the infant is not able to swallow at a rate to match the flow rate, the infant may compensate by allowing the milk to drool out of the mouth (Schrank, Al-Sayed, Beahm, \& Thach, 1998). If the infant is not able to swallow adequately to clear the fluid and does not compensate by drooling, residual milk remaining in the pharynx may place the infant at risk for aspiration (Rommel et al., 2011).

Healthy, full-term infants are generally able to coordinate the suck-swallow-breathe sequence quite well and able to tolerate the interruption in respiration by increasing respiratory rate and/or tidal volume during a pause in feeding (Bamford, Taciak, \& Gewolb, 1992). Medically fragile infants, particularly preterm infants with respiratory disease and those with cyanotic congenital heart disease, are often not able to tolerate this interruption in respiration and become physiologically unstable or discontinue oral feeding.

Infants who are medically fragile, such as those born preterm, with congenital heart disease, neurologic impairment, or other medical needs that require hospitalization beyond the typical post-partum hospitalization, must achieve a certain proficiency in oral feeding skill (or receive tube feedings) prior to discharge home. Bottle-feeding proficiency is typically established using bottle nipples available in the hospital (e.g., Similac or Enfamil brand nipples). Many nipples that are used for bottle-feeding in the hospital are not easily accessible by parents for use after hospital discharge and therefore, parents must choose a nipple to use at home. Medically fragile infants continue to develop oral feeding skills and coordination of swallowing and breathing after discharge (Kelly, Huckabee, Jones, \& Frampton, 2007; Vice \& Gewolb, 2008). Choice of nipple may be important for ensuring the safety of oral feeding and supporting the infant as oral feeding skills are developed.

Parents are faced with an overwhelming number of choices of bottle nipples and they often request input by their child's medical team in making this decision. Clinicians have little data to support parental guidance on choice of bottle nipple after discharge. Jackman (2013) reported findings of milk flow rates from 20 nipples that are available for purchase by parents in the community. However, this study was limited by the fact that only one nipple of each nipple type intended for repeated use was tested. This assumed that all nipples of the same type would be of comparable flow rate. She also reported on findings from bottle nipples intended for single-use only and found that when three nipples of each type were tested, there was wide variability in flow rates between nipples of the same type (Jackman, 2013). 
In our previous study on milk flow rates from bottle nipples used for feeding hospitalized infants (Pados, Park, Thoyre, Estrem, \& Nix, 2015), we improved upon the methods used by Jackman and tested more nipples of each type ( $n=10$, based on a power analysis). We found wide variability in milk flow rates of bottle nipples used in hospitals and found that within nipple type, there was considerable variation in milk flow rates for some brands. We also found that the name of the nipple provided little information about the actual flow rate of the nipple. The purpose of the current study was to test the milk flow rates and variability in flow rates of bottle nipples used after hospital discharge.

\section{Study Design and Methods}

No human or animal subjects were involved in this study, thus it was exempt from Institutional Review Board review. Purposeful sampling was used to select 26 nipple types that represented 15 common brands as well as variety in price per nipple and store location where sold (e.g., Babies R' Us, Walmart, Dollar Store) (Table 1). A power analysis was conducted on the first 80 nipples tested and revealed that 10 nipples of each nipple type was sufficient for comparing flow between nipple types with $80 \%$ power at an alpha of 0.05 , even if the effect size was $<.3$ and there was moderate correlation between nipples of the same type.

A standardized and controlled testing method was used to compare flow rates between nipple types (Pados, et al., 2015). This method was intended to test nipples under standardized conditions and was not intended to mimic milk flow rates achieved by an infant during oral feeding. We described the procedures in detail previously (Pados, et al., 2015). Briefly, a 60mL Grad-U-Feed Nurser (Mead Johnson \& Co, Glenview, IL) was used to test all nipples; nipples that did not fit on the Grad-U-Feed Nurser were tested on the bottle that was sold with the nipple. Similac Advance Stage 1 (20 calories/ounce) ready-to-feed formula (Abbott Laboratories, Abbott Park, IL) was used for all tests. The formula was changed every 10 tests to maintain consistency in the testing methods and to ensure that formula was not tested after being open for 30 minutes, which was a conservative approach given the manufacturer's recommendation to use within one hour of opening (Abbott Laboratories, Abbott Park, IL).

Approximately equal levels of hydrostatic pressure were maintained across all tests by maintaining a $2.5 \mathrm{~cm}$ height from the level of the liquid surface to the tip of the nipple. For example, standard-neck nipples that fit on the Grad-U-Feed Nurser required $50 \mathrm{~mL}$ of formula and the Dr. Brown's Wide-Neck bottle required $70 \mathrm{~mL}$ to achieve a height of 2.5 $\mathrm{cm}$.

A Medela Pump in Style Advanced breast pump (Medela, Inc., McHenry, IL) on the stimulation phase suction pattern was used to create a negative pressure system. The suction pressure from the breast pump was $180 \mathrm{mmHg}$. The bottle and nipple unit being tested were attached to a breast shield and held at a 30 degree angle. The negative pressure within the bottle was tested after every 50 tests using the Samba 201 Micro Pressure Measurement System (BIOPAC Systems, Inc., Goleta, CA) to ensure consistency across tests. Mean suction rate was 110 cycles/minute and mean negative pressure in the bottle was $14 \mathrm{mmHg}$. 
The weight of formula expressed in one minute was measured using a $500 \mathrm{~mL}$ beaker situated on a calibrated platform scale (Thermo Fisher Scientific, Inc, Waltham, MA), accurate to 0.01 grams. Tests were video-recorded and measurements confirmed by secondary review of the video tape. Milk flow rates $(\mathrm{mL} / \mathrm{min})$ were calculated using the density of Similac Advance formula of $0.97 \mathrm{~mL} / \mathrm{gram}$ (AVCalc, 2014). The Dr. Brown's nipples were tested with the venting system in place, which is how the nipple is intended to be used. The venting system comprises the cream colored vent insert and the blue vent reservoir (Handi-Craft Company, 2014).

\section{Statistical Analysis}

For each nipple type, the mean milk flow rate $(\mathrm{mL} / \mathrm{min})$ and standard deviation (SD) were calculated. The coefficient of variation (CV) was calculated as the SD divided by the mean. $\mathrm{CV}$ was used as a measure of variability in flow rates between the 10 nipples tested of the same nipple type, indicating the consistency of flow rates across nipples from the same nipple type. For the purposes of comparing variability between nipple types, $\mathrm{CV}$ was categorized into three levels: low $(<.1)$, moderate $(.1-.2)$, and high $(>.2)$.

All analyses were conducted in SAS version 9.3. An alpha of 0.05 was considered statistically significant, unless otherwise noted. Statistical comparisons were made separately for each brand using one-way ANOVA when normally distributed. The ShapiroWilk statistic was used to assess nipples for normality. When nipples were not normally distributed, non-parametric one-way ANOVA was used. Multiple comparison tests for the post-hoc analysis of one-way ANOVA utilized Duncan's multiple range test. When nonparametric one-way ANOVA was utilized, pairwise comparisons were made using the Wilcoxon Rank Sum Test (alpha was adjusted using a Bonferroni adjustment).

\section{Results}

\section{Mean Flow Rates}

Flow rates of bottle nipples available in the community setting for use after hospital discharge varied widely, with the slowest flow being the Avent Natural Newborn Flow (0+ months [mos], 1 hole) with a mean flow rate of $1.68 \mathrm{~mL} / \mathrm{min}$ and the fastest being the Dr. Brown's Standard Y-cut (9+ mos) with a mean flow rate of $85.34 \mathrm{~mL} / \mathrm{min}$ (Figure 1). Of the 26 nipples tested, 12 nipples had mean flow rates less than $10 \mathrm{~mL} / \mathrm{min}$ and nine nipples had flow rates between 10-20 mL/min. Only three nipples had mean flow rates between 20-30 $\mathrm{mL} / \mathrm{min}$ (Nuby Medium Flow Silicone Nipple [0+ mos], Medela Calma, and Fisher-Price Medium Flow) and only 2 nipples had mean flow rates greater than $30 \mathrm{~mL} / \mathrm{min}$ (Dr. Brown's Standard Level 3 [6+ mos] and Dr. Brown's Y-cut [9+ mos]).

\section{Variability in Flow}

There was wide variability between nipples types, from .03 for the Dr. Brown's Level 3 to . 37 for MAM Nipple 1 Slow Flow (0+ mos) (Figure 2). Eight of the nipple types had low CV (<.1), 9 nipple types had moderate CV (.1-.2), and nine nipple types had high CV (>.2). Five of the eight nipples that had low CV were from the Dr. Brown's brand. The other three with 
low CV were the Tommee Tippee Feeding Bottle Slow Flow (0+ mos) (.09), The First Years Breastflow Slow Flow (0-3 mos) (.06), and the Medela Calma (.05).

\section{Comparisons Within Brand}

Avent-Two nipples were tested from the Avent brand - the Avent Classic Newborn Flow ( $0+$ months, 1 hole) and Avent Natural Newborn Flow ( $0+$ months, 1 hole). The Avent Classic was significantly faster in flow rate than the Natural ( $8.19 \mathrm{vs} 1.67 \mathrm{~mL} / \mathrm{min} ; p<.01)$. The Avent Natural was the slowest of all 26 nipples tested in this study, but had high CV (. 25). The Avent Classic had moderate CV (.13).

Dr. Brown's-Six nipples were tested from the Dr. Brown's brand. Five of these were standard-neck nipples, including the Preemie (Premature infants), Level 1 ( $0+$ months), Level 2 ( $3+$ months), Level 3 (6+ months), and Y-cut (9+ months). The wide-neck Level 1 nipple ( $0+$ months) was also tested. All of the Dr. Brown's brand nipples were significantly different from one another $(p<.05)$, except for the Preemie and wide-neck Level 1, which were comparable ( 7.38 vs $7.82 \mathrm{~mL} / \mathrm{min} ; p=.08$ ). The Preemie and wide-neck Level 1 were the slowest, followed by the standard-neck Level 1 (9.21 mL/min), Level 2 (14.96), Level 3 (31.10), and the Y-cut (85.34). The Dr. Brown's Y-cut was the fastest of the 26 nipples tested in this study. All of the Dr. Brown's nipples had a low CV (.03 - .09), except for the Y-cut, which had a moderate CV (.13).

Evenflo-Three Evenflo brand nipples were tested - the Classic Slow Flow (0-3 months), Bebek Proflow Slow Flow (0-3 months), and the Purely Comfi Slow Flow (0-3 months). The Evenflo Classic and Bebek were comparable in flow rate $(12.91$ vs. $12.96 \mathrm{~mL} / \mathrm{min} ; p=$. $65)$ and were both significantly faster than the Purely Comfi $(8.62 \mathrm{~mL} / \mathrm{min} ; p<.01)$. All of the Evenflo brand nipples had a moderate CV $(.11-.16)$.

Medela-The Medela Wide Base Slow-Flow nipple and the Calma were tested. The Medela Calma was significantly faster than the Wide Base Slow-Flow (24.74 vs. $11.3 \mathrm{~mL} /$ min; $p<.01)$. The Calma had low CV (.05) and the Wide Base Slow-Flow had moderate CV (.16).

Playtex - Two nipples were tested from the Playtex brand - the VentAire Standard Slow Flow (0-3 months) and VentAire Wide Slow Flow (0-3 months). The VentAire Standard was significantly faster than the VentAire Wide (10.98 vs. $4.90 ; p<.01)$. CV was high (.24) for the Standard and moderate (.13) for the Wide.

The First Years-Two nipples were tested from The First Years brand - the Gumdrop Slim ( $0+$ months) and the Breastflow Slow Flow (0-3 months). The Gumdrop Slim was significantly faster than the Breastflow (11.14 vs. $8.08 \mathrm{~mL} / \mathrm{min} ; p<.01)$. The Gumdrop Slim had high CV (.24) while the Breastflow had low CV (.06).

\section{Clinical Nursing Implications}

Our study builds upon the recent study (Jackman, 2013) that has explored milk flow rates from bottle nipples available for feeding infants after discharge from the hospital. Our study 
has improved the methods to test flow rates of nipples and expanded the number of nipples tested. Our methods used, however, were limited in that only negative pressure (suction) was applied.

While many full-term, healthy infants are resilient feeders and may be able to manage differences in flow rates between nipple types, the extreme range in milk flow rates determined in this study may be significant for fragile infants being discharged. The name assigned to the nipple type does not provide clear information to parents attempting to choose a nipple that may be supportive of their fragile infant at the time of discharge. Most of the nipples tested were labeled "Slow" or "Newborn" or indicated on the packaging that they were intended for use in infants 0-3 months of age. However, within these categories, flow rates ranged from $1.68 \mathrm{~mL} / \mathrm{min}$ for the Avent Natural Newborn Flow to $15.12 \mathrm{~mL} / \mathrm{min}$ for the NUK Orthodontic Wide Slow Flow. Even within brand, the same flow label category did not result in comparable flow rate. For example, within the Avent brand, both the Avent Natural and Classic were labeled as "Newborn Flow (0+ months, 1 hole)," yet these nipples had significantly different flow rates. Similarly, within the Evenflo brand, all three nipples were labeled "Slow (0-3 months)," but had different flow rates.

The Medela Calma is an "all stage" nipple, which means that there is only one nipple sold with the Medela Calma system. This nipple is designed to achieve flow only when a certain level of sucking pressure is applied. We only tested the Medela Calma under one level of sucking pressure to maintain consistency across all tests, but it may be that the flow rate of this nipple type changes in a non-linear way as varying levels of sucking pressure are applied.

Variability in flow rates found between nipples of the same type may also be significant for fragile infants being discharged home. Nine of the 26 nipples tested in this study had a CV $>.2$, meaning that the flow rate from one nipple to the next within the same type varied by more than 20\%. Differences in manufacturing processes between brands and the methods used to create the nipple hole (e.g., punching a hole, laser cutting, or molding the nipple with a standard hole size) likely contribute to the variability within nipple type. Since it is common for households to use more than one nipple across a day, variability within the same type of nipple exposes the infant to changing conditions and decreases the predictability of feeding for the infant. Variability in flow rates means that from one feeding to the next, even if the same nipple type is used, the infant is exposed to different flow rates. Medically fragile infants may not be able to adapt to this variability (Scheel, Schanler, \& Lau, 2005). Choosing a nipple that is consistent in the flow rate delivered may be more supportive of the infant's ability to learn and develop oral feeding skills.

Nipples for this study were purposefully chosen to reflect variation in price per nipple and store location sold in order to make these findings useful to parents of varying socioeconomic status. Clinicians caring for fragile infants can use this information to guide parents in selecting a nipple for use at home that will be supportive of the safety and success of the infant's oral feeding. In choosing a nipple, both the flow rate and the variability in flow should be taken into consideration. Starting with a nipple that is truly slow flow $(<10$ $\mathrm{mL} / \mathrm{min}$ ) and has low variability $(<.1)$, then advancing as tolerated is likely the safest 
progression for very fragile infants. It should be noted that tightening the nipple ring to the bottle too tightly interferes with pressure venting and may cause the nipple to collapse, which is sometimes interpreted as the flow being too slow. The infant should be given the opportunity to feed with the chosen nipple while they remain hospitalized to ensure the infant's ability to safely and effectively feed.

\section{Acknowledgments}

Sources of Funding: This study was funded by a Sigma Theta Tau International Honor Society of Nursing Alpha Alpha Chapter Research Award and was supported by the National Institute of Nursing Research of the National Institutes of Health under Award Number 5F31NR011262 (Pados). Medela Inc. provided Medela Calma nipples for testing.

\section{References}

al-Sayed LE, Schrank WI, Thach BT. Ventilatory sparing strategies and swallowing pattern during bottle feeding in human infants. Journal of Applied Physiology. 1994; 77(1):78-83. Retrieved from http://jap.physiology.org/. [PubMed: 7961278]

AVCalc. Food weight to volume conversions. 2014. Retrieved 5/18/2014, 2014 from http://www.aquacalc.com/calculate/food-weight-to-volume

Bamford O, Taciak V, Gewolb IH. The relationship between rhythmic swallowing and breathing during suckle feeding in term neonates. Pediatric Research. 1992; 31(6):619-624. DOI: 10.1203/00006450-199206000-00016 [PubMed: 1635825]

Barlow SM. Oral and respiratory control for preterm feeding. Current Opinions in Otolaryngology \& Head and Neck Surgery. 2009; 17(3):179-186. DOI: 10.1097/MOO.0b013e32832b36fe

Handi-Craft Company. How They Work: Bottles. 2014. Retrieved July 8, 2014 from http:// www.drbrownsbaby.com/bottles-accessories/how-they-work

Jackman KT. Go with the flow: Choosing a feeding system for infants in the neonatal intensive care unit and beyond based on flow performance. Newborn \& Infant Nursing Reviews. 2013; 13:31-34. DOI: 10.1053/j.nainr.2012.12.003

Kelly BN, Huckabee ML, Jones RD, Frampton CM. The early impact of feeding on infant breathingswallowing coordination. Respiratory Physiology \& Neurobiology. 2007; 156(2):147-153. DOI: 10.1016/j.resp.2006.09.007 [PubMed: 17101302]

Mathew OP. Breathing patterns of preterm infants during bottle feeding: Role of milk flow. Journal of Pediatrics. 1991; 119(6):960-965. DOI: 10.1016/S0022-3476(05)83056-2 [PubMed: 1960618]

Pados BF, Park J, Thoyre SM, Estrem H, Nix WB. Milk flow rates from bottle nipples used for feeding hospitalized infants. American Journal of Speech-Language Pathology. 2015; Advance online publication. doi: 10.1044/2015_AJSLP-15-0011

Rommel N, Van Wijk M, Boets B, Hebbard G, Haslam R, Davidson G, Omari T. Development of pharyngo-esophageal physiology during swallowing in the preterm infant. Neurogastroenterology \& Motility. 2011; 23:e401-e408. [PubMed: 21827583]

Scheel CE, Schanler RJ, Lau C. Does the choice of bottle nipple affect the oral feeding performance of very-low-birthweight (VLBW) infants? Acta Pediatrica. 2005; 94(9):1266-1272.

Schrank W, Al-Sayed LE, Beahm PH, Thach BT. Feeding responses to free-flow formula in term and preterm infants. Journal of Pediatrics. 1998; 132(3 Pt 1):426-430. DOI: 10.1016/ S0022-3476(98)70014-9 [PubMed: 9544895]

Vice FL, Gewolb IH. Respiratory patterns and strategies during feeding in preterm infants. Developmental Medicine and Child Neurology. 2008; 50(6):467-472. DOI: 10.1111/j. 1469-8749.2008.02065.x [PubMed: 18422676] 


\section{Callouts}

1. Milk flow rate is an important characteristic of bottle nipples to consider when guiding parents in choosing a nipple for use after hospital discharge.

2. Little information is available to clinicians to inform parental guidance.

3. Milk flow rates of bottle nipples available for use after hospital discharge varied widely and within a single nipple type, there was considerable inconsistency.

4. The label on a bottle nipple is not an accurate indicator of the flow rate for an individual nipple.

5. Clinicians should consider flow rate and variability when guiding parents to choose a bottle nipple for use after discharge. 


\section{Suggested Clinical Nursing Implications}

- Milk flow rate and variability in flow should both be considered when guiding parents to choose a nipple for bottle-feeding after discharge.

- The name on the label of a nipple does not provide clear information about the flow rate of the nipple.

- Infants should be encouraged to transition to the nipple that will be used at home while they remain hospitalized to ensure that they can safely and effectively feed. 


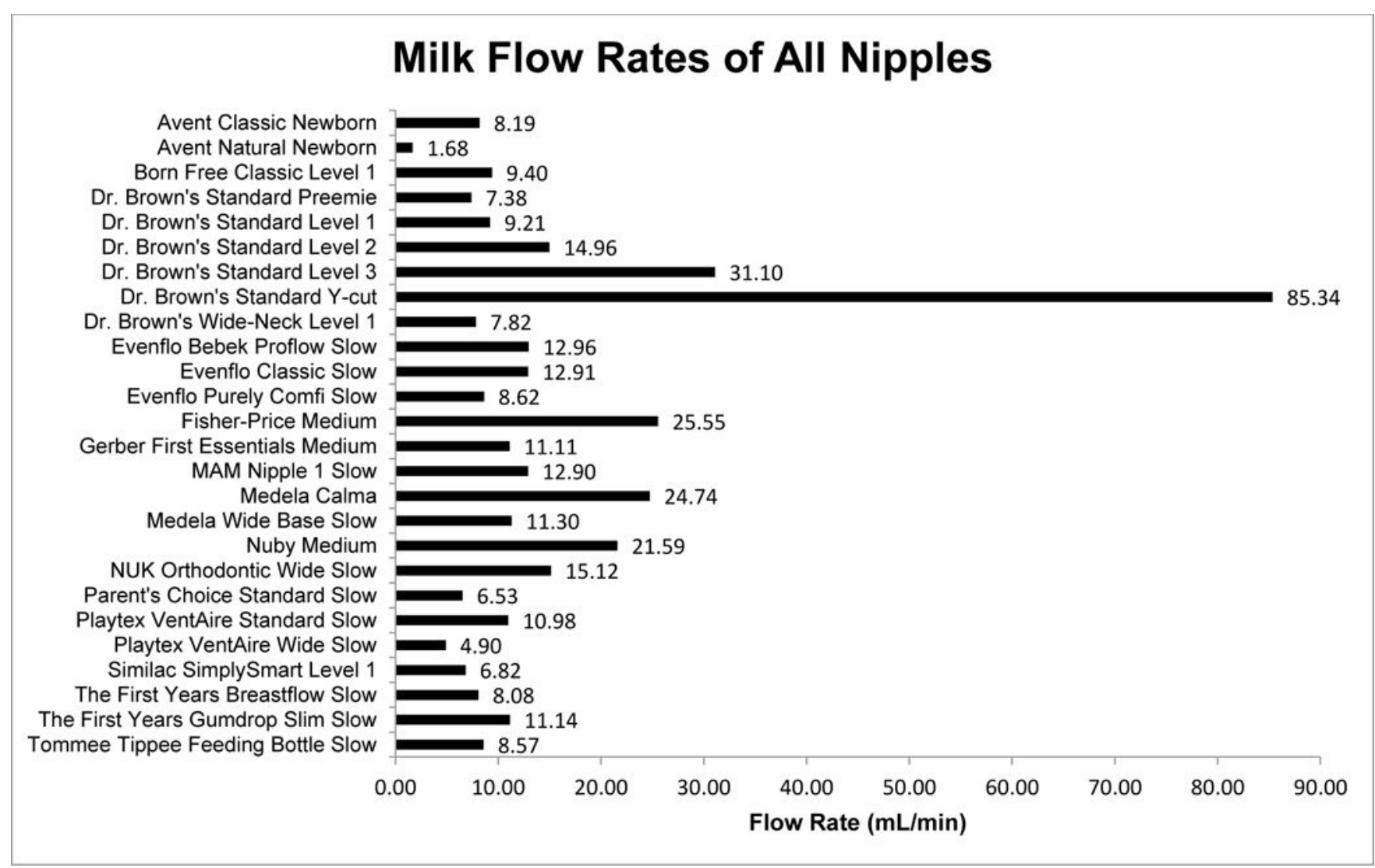

Figure 1.

Milk Flow Rates of All Nipples Tested 


\section{Coefficient of Variation of Milk Flow Rates}

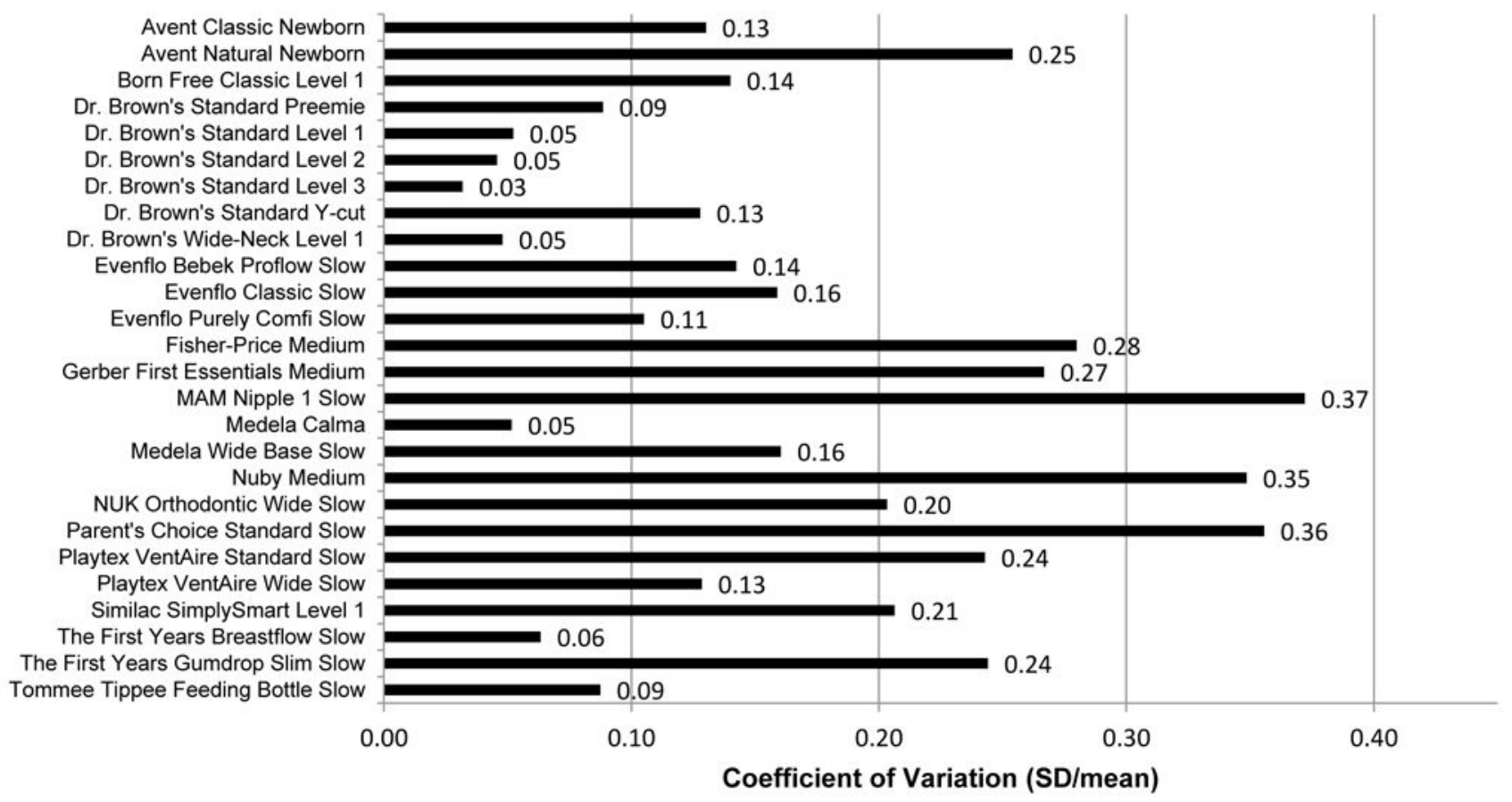

Figure 2.

Coefficient of variation (CV) of milk flow rates calculated as the standard deviation (SD) divided by mean flow rate of nipples tested of the same type $(n=10)$. CV $<.1=$ low, CV $0.1-$ $0.2=$ moderate, $\mathrm{CV}>0.2=$ high . 
Table 1

Nipples Tested, Flow Rates, and Coefficient of Variation (CV)

\begin{tabular}{|c|c|c|c|c|}
\hline Brand & Manufacturer & Nipples Tested and Label Information & $\begin{array}{l}\text { Mean (Range) Flow } \\
\text { Rate in } \mathrm{mL} / \mathrm{min}\end{array}$ & CV \\
\hline Avent & Philips Avent & $\begin{array}{l}\text { Classic Newborn Flow }(0+\text { mos, } 1 \text { hole }) \\
\text { Natural Newborn Flow }(0+\text { mos, } 1 \text { hole })\end{array}$ & $\begin{array}{c}8.19(6.62-10.25) \\
1.68(1.15-2.57)\end{array}$ & $\begin{array}{l}.13 \\
.25\end{array}$ \\
\hline Born Free & Born Free & Classic Level 1 (Slow-Flow, 0-3 mos) & $9.40(6.98-12.21)$ & .14 \\
\hline Dr. Brown's & Handi-Craft Co. & $\begin{array}{l}\text { Standard Preemie Flow (Premature) } \\
\text { Standard Level } 1(0+\text { mos }) \\
\text { Standard Level } 2(3+\text { mos }) \\
\text { Standard Level } 3(6+\text { mos }) \\
\text { Standard Y-cut }(9+\text { mos }) \\
\text { Wide-Neck Level } 1(0+\text { mos })\end{array}$ & $\begin{array}{c}7.38(6.15-8.21) \\
9.21(8.42-9.90) \\
14.96(13.75-15.80) \\
31.10(29.25-32.19) \\
85.34(70.00-108.10) \\
7.82(7.14-8.31)\end{array}$ & $\begin{array}{l}.09 \\
.05 \\
.05 \\
.03 \\
.13 \\
.05\end{array}$ \\
\hline Evenflo & Evenflo Feeding & $\begin{array}{l}\text { Bebek Proflow Slow Flow }(0-3 \text { mos }) \\
\text { Classic Slow Flow ( } 0-3 \text { mos }) \\
\text { Purely Comfi Slow Flow (0-3 mos) }\end{array}$ & $\begin{array}{c}12.96(11.02-16.11) \\
12.91(7.79-15.56) \\
8.62(7.69-10.07)\end{array}$ & $\begin{array}{l}.14 \\
.16 \\
.11\end{array}$ \\
\hline Fisher-Price & Fisher-Price & Medium Flow & $25.55(17.48-41.42)$ & .28 \\
\hline Gerber First Essentials & Nestlé & Silicone Nipple Medium Flow (4 mos+) & $11.11(8.05-17.29)$ & .27 \\
\hline MAM & MAM USA & Nipple 1 Slow Flow $(0+$ mos $)$ & $12.90(8.75-21.35)$ & .37 \\
\hline Medela & Medela, Inc. & $\begin{array}{l}\text { Calma } \\
\text { Wide Base Slow Flow }\end{array}$ & $\begin{array}{c}24.74(22.99-26.50) \\
11.30(8.63-14.12)\end{array}$ & $\begin{array}{l}.05 \\
.16\end{array}$ \\
\hline Nuby & Nuby Inc. & Medium Flow Silicone Nipple $(0+$ mos $)$ & $21.59(8.57-33.23)$ & .35 \\
\hline NUK & NUK USA, LLC & Orthodontic Wide Slow Flow (0-6 mos) & $15.12(11.99-22.90)$ & .20 \\
\hline Parent's Choice & Wal-Mart, Inc. & Standard Slow Flow $(0+$ mos $)$ & $6.53(1.62-8.26)$ & .36 \\
\hline Playtex & Playtex Products, LLC & $\begin{array}{l}\text { VentAire Standard Slow Flow }(0-3 \text { mos }) \\
\text { VentAire Wide Slow Flow }(0-3 \text { mos })\end{array}$ & $\begin{array}{c}10.98(6.56-16.54) \\
4.90(3.96-5.78)\end{array}$ & $\begin{array}{l}.24 \\
.13\end{array}$ \\
\hline Similac & Abbott Nutrition & SimplySmart Level 1 & $6.82(5.32-10.21)$ & .21 \\
\hline The First Years & TOMY Intl. Inc. & $\begin{array}{l}\text { Breastflow Slow Flow (0-3 mos) } \\
\text { Gumdrop Slim (0 mos }+)\end{array}$ & $\begin{array}{c}8.08(7.30-9.16) \\
11.14(6.95-15.79)\end{array}$ & $\begin{array}{l}.06 \\
.24\end{array}$ \\
\hline Tommee Tippee & Mayborn USA & Feeding Bottle Slow Flow $(0+$ mos $)$ & $8.57(7.54-9.87)$ & .09 \\
\hline
\end{tabular}

Note. $\mathrm{CV}=$ coefficient of variation; Inc. $=$ incorporated; Intl. $=$ international $\mathrm{mL}=$ milliliters mos $=$ months 